\title{
Enhancing the Utility of the Dexamethasone Suppression Test: A Chart Review and Application of Bayes' Theorem
}

\author{
Scott B. Patten, M.D. \\ Calgary General Hospital, Calgary Alberta
}

Follow this and additional works at: https://jdc.jefferson.edu/jeffjpsychiatry

Part of the Psychiatry Commons

Let us know how access to this document benefits you

\section{Recommended Citation}

Patten, M.D., Scott B. (1990) "Enhancing the Utility of the Dexamethasone Suppression Test: A Chart Review and Application of Bayes' Theorem," Jefferson Journal of Psychiatry. Vol. 8 : Iss. 1 , Article 9. DOI: https://doi.org/10.29046/JJP.008.1.004

Available at: https://jdc.jefferson.edu/jeffjpsychiatry/vol8/iss1/9

This Article is brought to you for free and open access by the Jefferson Digital Commons. The Jefferson Digital Commons is a service of Thomas Jefferson University's Center for Teaching and Learning (CTL). The Commons is a showcase for Jefferson books and journals, peer-reviewed scholarly publications, unique historical collections from the University archives, and teaching tools. The Jefferson Digital Commons allows researchers and interested readers anywhere in the world to learn about and keep up to date with Jefferson scholarship. This article has been accepted for inclusion in Jefferson Journal of Psychiatry by an authorized administrator of the Jefferson Digital Commons. For more information, please contact: JeffersonDigitalCommons@jefferson.edu. 


\title{
Enhancing the Utility of the Dexamethasone Suppression Test: A Chart Review and Application of Bayes' Theorem
}

\author{
Scott B. Patten M.D.
}

\section{INTRODUCTION}

The dexamethasone suppression test (DST) is the most extensively studied biological test in psychiatry. Despite this, its role in the diagnostic assessment of psychiatric patients remains controversial. Shortcomings of the test include limited sensitivity (45\%) and limited specificity (75-80\%) (1). The DST has many proposed uses, including the differentiation of endogenous from non-endogenous depressions, helping to decide when maintenance medications may be withdrawn, and as a diagnostic test for major depression. This paper is concerned with the latter use only, that is, the ability of the DST to function as a useful diagnostic test for major depression.

The significance of the low sensitivity and specificity of the DST have been discussed extensively in the psychiatric literature $(2,3)$. When the DST is used as a diagnostic test for major depression its sensitivity represents the likelihood of a positive test given that the tested patient has a major depression. Specificity represents the likelihood of a negative test given that the tested patient does not have a major depression. Notably, both sensitivity and specificity represent probabilities conditional on the presence or absence of disease. However, in the usual clinical situation, the diagnosis is unknown at the time the diagnostic test is ordered. Indeed, if the diagnosis is known then the test should not be performed. Thus neither sensitivity nor specificity is directly relevant to the interpretation of the results of diagnostic tests in a clinical setting. Another parameter, the positive predictive value $(+\mathrm{PV})$, is of more clinical relevance.

The usual formula for $+\mathrm{PV}$ is:

$$
+\mathrm{PV}=\frac{\text { true positives }}{\text { true positive }+ \text { false positives }}
$$

This formula is difficult to apply in practice because the 'true' disease status must be known to identify true and false positives. However, the $+\mathrm{PV}$ can be estimated using another formula (which is actually an application of Bayes' theorem):

$$
+\mathrm{PV}=\frac{\mathrm{Se}(\mathrm{Pr})}{\mathrm{Se}(\mathrm{Pr})+(1-\mathrm{Sp})(1-\mathrm{Pr})}
$$


Where:

$$
\begin{aligned}
& \mathrm{Se}=\text { sensitivity } \\
& \mathrm{Sp}=\text { specificity } \\
& \mathrm{Pr}=\text { pre-test probability }
\end{aligned}
$$

The + PV of the DST represents the probability that a patient has major depression, given that the DST is nonsuppressing. Thus, unlike sensitivity and specificity, + PV quite closely reflects the process of clinical judgement faced by the clinician in interpreting the results of a diagnostic test such as the DST. The $+\mathrm{PV}$ is affected by sensitivity, specificity and by the pre-test probability. Pre-test probability refers to the likelihood that a patient has the condition being tested for before the diagnostic test is performed. The usefulness of any diagnostic test relates to its ability to change this probability. In an absolute sense, the probability that the relevant illness is present is not changed by the diagnostic test. However, a clinician's best judgement about the correct diagnosis can be changed by a test result. For example, if a clinician believes that the probability of major depression in a given patient is say, 50\% (pre-test probability) and a positive DST increases that probability to $90 \%$ (in other words has a positive predictive value of $90 \%$ ) then the test has obviously been very useful as a diagnostic test for that patient.

The pre-test probability of major depression in a given patient is influenced by the clinical symptomatology exhibited by that patient. This parameter is difficult to quantify. However, the pre-test probability is also influenced by a number of demographic variables which are easy to quantify such as age, sex, etc. The pre-test probability of depression in any subgroup of patients identified by such a variable (eg. females aged 40-60 years) is equivalent to the point prevalence of major depression in that particular subgroup of patients. Thus, prevalence rates of major depression in subgroups of patients in a clinical setting allow for the estimation of pre-test probability and subsequently, the use of this parameter in clinical decision making.

The relationship between predictive value, sensitivity, specificity and pretest probability is of critical importance in the interpretation of diagnostic test results. For example, the DST is generally regarded as a relatively specific test if certain exclusion criteria are adhered to in choosing candidates for the test. By definition, a specific test is very likely to be negative if a patient does not have the condition being tested for, in this case, depression. Clinicians, therefore, may assume that a positive DST is fairly strongly indicative of depression. However, this is not necessarily the case; in fact, for statistical reasons (which will be discussed below) a positive DST may be very significant in some cases and virtually insignificant in others.

As discussed previously, the usefulness of any diagnostic test relates to its ability to alter the pre-test probability. The statistical method designed to quantify this alteration in probability is the post-test probability difference. This statistic represents the difference between the pre- and post-test probability 
which is produced by a positive test. The post-test probability difference is perhaps the best estimate of the contribution (in terms of probability) of a diagnostic test to clinical judgement.

Another issue related to the interpretation of diagnostic tests is the negative predictive value $(-\mathrm{PV})$. This parameter quantifies the probability that an individual with a negative test actually does not have the disease in question. Because the DST is an insensitive test, its negative predictive value is always very small and negative test results can never play a major role in diagnostic decision making. The negative predictive value of the DST will therefore not be discussed.

Problems related to the interpretation of diagnostic data, such as those discussed above, have received attention in the medical literature (4), including the psychiatric literature (5). On some occasions, these principles have been applied formally to complex clinical problems $(6,7)$. However, neither empirically derived estimates of $+\mathrm{PV}$ nor of post-test probability difference are generally available in the psychiatric literature. In fact, because the $+\mathrm{PV}$ varies depending on the pre-test probability of depression, the + PV of the DST is likely to be different in different groups and in different clinical settings. Using Bayes' theorem and estimates of sensitivity, specificity, and pre-test probability of depression, one can calculate $+\mathrm{PV}$ and post-test probability difference. Because pre-test probability can be easily estimated from chart reviews or existing records, this method provides a practical way of making these parameters available to clinicians in a particular setting who may use the DST. In fact, the use of Bayes' Theorem should enable any clinician with a basic knowledge of the patient population in his or her clinical setting to estimate the predictive value of the DST in a few seconds using only basic algebra. This, in turn, should result in more sophisticated and accurate interpretation of DST results.

ESTIMATION OF PREDICTIVE VALUE OF THE DST:

As stated previously, Bayes' theorem can be written as:

$$
+\mathrm{PV}=\frac{\mathrm{Se}(\mathrm{Pr})}{\mathrm{Se}(\mathrm{Pr})+(1-\mathrm{Sp})(1-\mathrm{Pr})}
$$

Where:

$$
\begin{aligned}
& \mathrm{Se}=\text { sensitivity } \\
& \mathrm{Sp}=\text { specificity } \\
& \operatorname{Pr}=\text { pre-test probability }
\end{aligned}
$$

For any particular subgroup of psychiatric patients in a particular setting the pre-test probability of depression can be estimated as the prevalence of major depression in that subgroup. The necessary information can be easily gathered by examining existing records of samples of patients in that facility. In some cases it may be possible to gather the necessary information from adminis- 
trative statistical records or medical records data bases. For example, if $40 \%$ of females admitted to a particular unit have major depression, then the pre-test probability of major depression for females, as a group, is $40 \%$. Existing estimates of sensitivity and specificity from the current literature can be used in the formula. The post-test probability difference is calculated by subtracting the pre-test probability from the $+\mathrm{PV}$.

As an example of this method, we have recently estimated the $+\mathrm{PV}$ and post-test probability difference of the DST in various subgroups of patients admitted to the Calgary General Hospital. Within this paper, the example serves two purposes; it generates estimates of $+\mathrm{PV}$ and post-test probability difference for the DST (such estimates are generally not available in the psychiatric literature), and it also illustrates the method whereby such estimates can be generated for different psychiatric settings using Bayes' Theorem.

\section{METHODS}

The medical records of all patients discharged from the psychiatric inpatient units at the Calgary General Hospital over a three month period were reviewed. This sample consisted of 243 patients. Information regarding the presence or absence of a number of variables relevant to depression was gathered. The variables included age, sex, marital status, employment status, past psychiatric history, family history, and the presence or absence of suicidal ideation. The patients in the sample were divided according to these variables into sub-groups and the proportion of patients in each group receiving a clinical diagnosis of major depression was recorded. The prevalence of depression in each subgroup was taken as the pre-test probability of depression in that subgroup. Bayes' theorem was then used to estimate the + PV for the DST in each group. Previously published estimates of sensitivity and specificity (1) were used in the calculations. Post-test probability difference was calculated by subtracting the pre-test probability from the $+\mathrm{PV}$.

\section{RESULTS}

The results of the chart review are presented in two stacked bar graphs, table \#1 and table \#2. In each graph, the bottom portions of the bars represent the pre-test probability of depression in the variously defined clinical groups. The upper portions represent the amount the DST adds to the estimated probability of major depression (the post test probability difference). The total height of each bar represents the positive predictive value of the test.

Within different clinical groups, the pre-test probability of depression varies markedly. In this chart review, the prevalence of major depression varied from $3 \%$ (in males aged 20-40) to 59\% (individuals with a past history of depression). It can be seen that the post-test probability of depression is larger for clinical 
TABLE 1.

Predictive Value of the DST

Pre \& Post-Test Probability by Age \& Sex

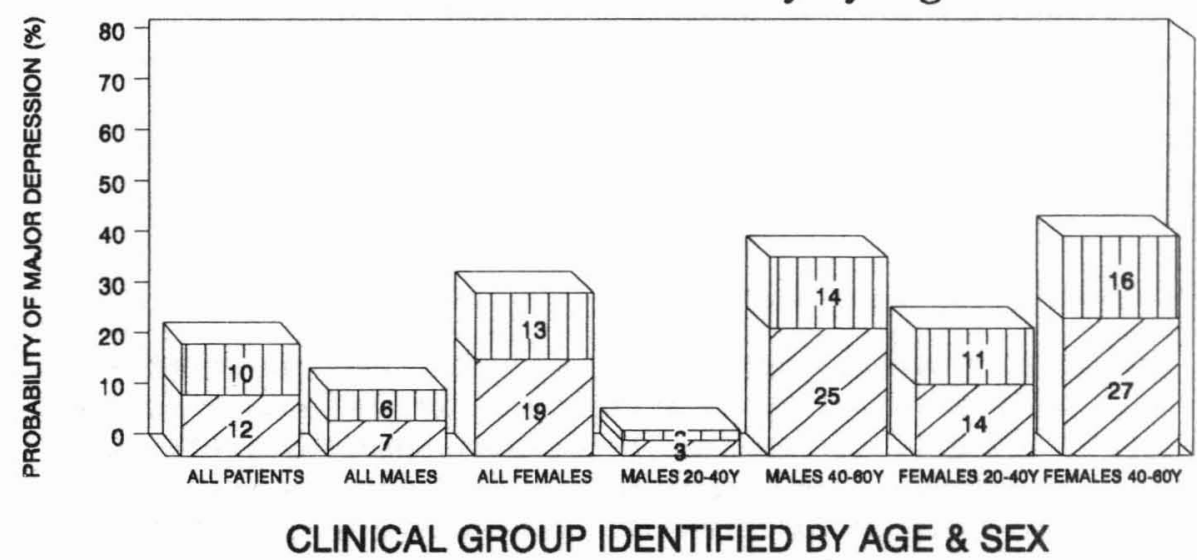

$\square$ PRE-TEST $\square$ POST-TEST

TABLE 2.

Predictive Value of the DST

For Different Clinical Groups

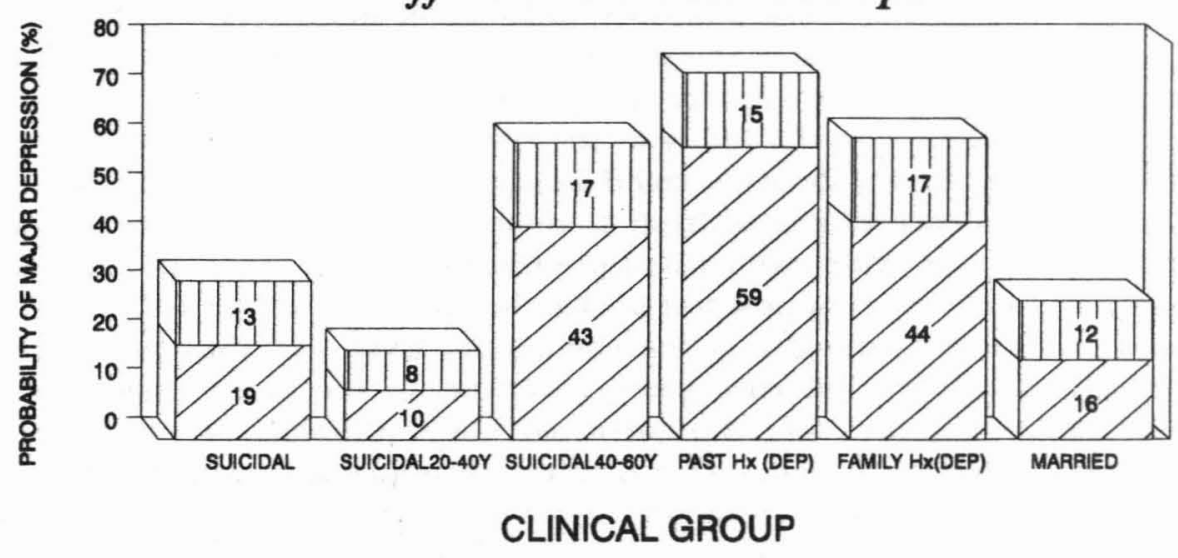

$\square$ PRE-TEST $\square$ POST-TEST 
groups with a higher pre-test probability of depression. In reality, the post-test probability difference is maximal when the pre-test probability is $50 \%$. The post-test probability, consequently, tends to be larger in groups with high pre-test probability.

Generally, the test generated higher predictive values in 40-60 year olds than in 20-40 year olds. The test generated higher predictive values in patients belonging to clinical groups which are at high risk for depression; females, individuals with a past history of depression, and individuals with a family history of depression.

\section{DISCUSSION}

In several situations, namely in patients with a family history of depression and in patients with a past history of depression the DST generated reasonably high + PV (61\% and $74 \%$ respectively) and post-test probability differences (15\% and $17 \%$ respectively). This suggests that the DST might be of some diagnostic value in these situations. Of course the DST will not be useful for all patients with a family history of depression or a past history of depression. Many such patients will be diagnosable on clinical grounds alone; laboratory tests are not helpful in this group. However, it is reasonable to assume that some patients in these subgroups will present enough diagnostic uncertainty that a diagnostic test could be helpful. In the other subgroups of patients which were identified, the predictive value and post-test probability difference of the test was so low that it is unlikely that the DST could be of any benefit to these patients.

These estimates of $+\mathrm{PV}$ apply only to one clinical population at one point in time. The usefulness of the DST, as measured by + PV and post-test probability difference, might vary with time at a particular institution and certainly would be expected to vary between institutions. However, Bayes' Theorem can be applied with minimal expense and effort to aid in the interpretation of the DST.

The strategy described in this paper involves the collection of data relevant to the application of Bayes' theorem in an institution, and the subsequent use of the theorem to enhance the utility of the test in that institution. It should be mentioned that Bayes' theorem could be applied to other sorts of data. For example, prevalence estimates from community based epidemiologic studies could be used to provide estimates of pre-test probability. Such an application would lead to estimates of post-test probability and post-test probability difference which were meaningful at the community level, and thus have a more intuitively meaningful interpretation. However, to the extent that selective factors leading to admission at a particular hospital define the nature of the population at that institution, such estimates may not be applicable clinically.

In summary, relative to the interpretation of some other diagnostic tests, the interpretation of the DST is difficult. The concepts of sensitivity and specificity may not provide clear guidance for clinical decision making when the results of the test become available. Concepts such as positive predictive value 
and post-test probability difference can be very helpful in capturing the true implications of DST results. Bayes' theorem provides a method of estimating these parameters. The method involves gathering some data relevant to the clinical population in which the test is to be interpreted and some simple algebraic calculations.

\section{REFERENCES}

1. The APA Task Force on Laboratory Tests in Psychiatry: The dexamethasone suppression test: an overview of its current status in psychiatry. Am J Psychiatry 144(10): 1253-1262, 1987

2. Schuman SH, Malcolm RM, Hainer BL: Dexamethasone suppression test and diagnosis of melancholia (letter). Arch Gen Psychiatry 39:1218, 1982

3. Young MJ: Dexamethasone suppression test and diagnosis of melancholia (letter). Arch Gen Psychiatry 39:1218-1219, 1982

4. Patton DD: Introduction to clinical decision making. Semin Nucl Med 8(4):273-282, 1978

5. Baldessarini RJ, Finklestein S, Arana GW: The predictive power of diagnostic tests and the effect of prevalence of illness. Arch Gen Psychiatry 40:569-573, 1983

6. Hamilton GW, Trobaugh GB, Ritchie JL, Gould KL, DeRouen TA, Williams DL: Myocardial imaging with 201 thallium: An analysis of clinical usefulness based on Bayes' theorem. Semin Nucl Med 8(4):358-364, 1978

7. Vea HW, Sirotta PS, Nelp WB: Ventilation-perfusion scanning for pulmonary embolism: refinement of predictive value through an application of Bayes' theorem. AJR 145:967-972, 1985 